\title{
FABRIQUE DES ARCHIVES, FABRIQUE DE L'HISTOIRE DU MOYEN ÂGE AU XIX SIÈCLE UNE BIBLIOGRAPHIE
}

\author{
Olivier PONCET
}

La présente bibliographie s'inscrit dans la logique de réflexions exposée dans la présentation (p. 1-14). Elle est d'abord indicative et n'a aucune prétention un tant soit peu exhaustive. Classée méthodiquement et par ordre de production des ouvrages ou articles, elle répond quelquefois à des choix personnels. L'accent a été mis, en particulier, sur des contributions figurant dans des revues professionnelles souvent peu consultées par les chercheurs en sciences humaines. Lorsqu'un titre rend mal le propos de la contribution ou masque une prise de position affirmée (au-delà de la simple exposition d'un problème ou de ses solutions), un léger commentaire, entre crochets carrés, suit la mention d'un titre.

Un index des noms d'auteurs permet de pallier les effets négatifs de la répartition des titres selon un ordre méthodique.

Il faut souhaiter que le constat de lacunes apparentes sur quelques sujets essentiels puisse stimuler les recherches : seraient ainsi mieux connus l'histoire des archives en France depuis 1880 environ, l'histoire des législations archivistiques françaises du $\mathrm{XX}^{\mathrm{e}}$ siècle, les archives locales sous l'Ancien Régime ou encore les emprunts et exportations de modèles archivistiques à l'échelle européenne puis mondiale.

1. Histoire des archives

1.1. Généralités

1.2. Archives dans l'Antiquité

1.3. Archives en France

1.3.1. Archives en France au Moyen Âge

1.3.2. Archives en France sous l'Ancien Régime

1.3.3. Archives en France après 1789

2. Archivistique

2.1. Généralités

2.1.1. Bibliographie

2.1.2. Revues

Revue de synthèse : 5érie, année 2004, p. 183-195. 


\subsection{Ouvrages et manuels}

2.3. Respect des fonds et traitement des archives

2.4. Confection des instruments de recherche

3. Épistémologie, historiographie et archives

3.1. Numéros spéciaux de revues

3.2. Autres travaux

\section{HISTOIRE DES ARCHIVES}

\subsection{Généralités}

1. Pistolese (Serafino), «Développement et caractère des archives du onzième siècle à nos jours. Essai historique », Archivi d'Italia, 2e sér., t. I, 1933-1934, p. 251-298. [Réimpr. Les Archives européennes du onzième siècle à nos jours. Essai historique et juridique, Rome, Annales Institutorum, Biblioteca d'arte editrice, 1934 (Bibliothèque des Annales Institutorum, suppl., 4).]

2. SANDRI (Leopoldo), «La storia degli archivi », Rassegna degli Archivi di Stato, t. XVIII, 1958, p. 109-134. [Résumé et repris dans Archivum, t. XVIII, 1968, p. 101-113.]

3. FAVIER (Jean), Les Archives, Paris, Presses universitaires de France, 1959, $7 \mathrm{e}$ éd. Paris, Presses universitaires de France, 2001 (Que sais-je ?, 805).

4. BAUTIER (Robert-Henri), Les Archives, in SAMARAN (Charles), dir., L'Histoire et ses méthodes, Paris, Gallimard, 1961 (Encyclopédie de la Pléiade, 11), p. 1120-1167.

5. D'AdDARIO (Arnaldo), «Lineamenti di storia dell'archivistica (secc. XVI-XIX)», Archivio Storico Italiano, fasc. 543, 1990, p. 3-35.

6. Histoire et archives : revue semestrielle, Paris, Champion, depuis 1997. [Soutenue par la Société des amis des Archives de France.]

7. Coeuré (Sophie) et Duclert (Vincent), Les Archives, Paris, La Découverte, 2001 (Repères).

\subsection{Archives dans l'Antiquité}

8. POSNER (Ernst), Archives in the ancient world, Cambridge, MA, Harvard University Press, 1972.

9. Goody (Jack), The Logic of writing and the organization of society, CambridgeNew York, Cambridge University Press, 1986, trad. franç., La Logique de l'écriture. Aux origines des sociétés humaines, Paris, Armand Colin, 1986.

10. Demougin (Ségolène), éd., La Mémoire perdue. À la recherche des archives oubliées, publiques et privées, de la Rome antique, Paris, Publications de la Sorbonne, 1994 (Histoire ancienne et médiévale, 30).

11. Sickinger (James P.), Public records and archives in classical Athens, Chapel Hill, University of North Carolina Press, 1999 (Studies in the History of Greece and Rome). 


\subsection{Archives en France}

12. Pomian (Krzysztof), «Les Archives. Du Trésor des chartes au Caran », in NorA (Pierre) et al., dir., Les Lieux de mémoire. Vol. III : Les France, 3 : De l'archive à l'emblème, Paris, Gallimard, 1993 (Bibliothèque illustrée des histoires), p. 192-200.

13. HiLdESHEIMER (Françoise), Les Archives de France, mémoire de l'histoire, Paris, Honoré Champion, 1997 (Histoire et archives, hors-série, 1).

\subsubsection{Archives en France au Moyen Âge}

14. Delaborde (Henri François), Étude sur la constitution du Trésor des chartes et sur les origines de la série des sacs dite aujourd'hui supplément du Trésor des chartes. [Introduction à] Layettes du Trésor des chartes, t. V, Paris, Plon, 1909, p. I-CCXXIV. [Synthèse d'une dizaine d'études de l'auteur parues depuis 1897.]

15. Richard (Jean), «Les archives et les archivistes des ducs de Bourgogne dans le ressort de la chambre des comptes de Dijon », Bibliothèque de l'École des chartes, t. CV, 1944, p. 123-169.

16. RÜCK (Peter), «Die Ordnung der herzoglich-savoyischen Archive unter Amadeus VIII (1398-1451)», Archivalische Zeitschrift, 67, 1971, p. 11-101, trad. ital. augm., L'Ordinamento degli archivi ducali di Savoia sotto Amadeo VIII, 1398-1451, Rome, 1977, 156 p. (Quaderni della Rassegna degli Archivi di Stato, 48).

17. Contamine (Philippe), «La mémoire de l'État. Les archives de la Chambre des comptes du roi de France à Paris au Xve siècle », in Media in Francia, recueil de mélanges offerts à Karl-Ferdinand Werner, Maulévrier, Paris, Institut historique allemand, 1989, p. 85-100, repr. in ConTAMINE (Philippe), Des pouvoirs en France, 1300-1500, Paris, Presses de l'École normale supérieure, 1992, p. 237-250.

18. Sот (Michel), Un historien et son Église. Flodoard de Reims, Paris, Fayard, 1993.

19. MAtTÉONI (Olivier), « La conservation et le classement des archives dans les chambres des comptes de la principauté bourbonnaise à la fin du Moyen Âge », in Contamine (Philippe) et MATtÉONi (Olivier), dir., La France des principautés. Les chambres des comptes (XIVe et XV siècles), colloque tenu [...] à MoulinsYzeure les 6, 7 et 8 avril 1995, Paris, Comité pour l'histoire économique et financière de la France, 1996 (Histoire économique et financière de la France. Animation de la recherche), p. 65-81.

20. GuYOTJEANNIN (Olivier), « Super omnes thesauros rerum temporalium. Les fonctions du Trésor des chartes du roi de France (XIV $-\mathrm{XV}^{\mathrm{e}}$ siècles) », in FIANU (Kouky) et GuTH (Daniel J.), éd., Écrit et pouvoir dans les chancelleries médiévales. Espace français, espace anglais, actes du colloque de Montréal, 1995, Louvain-la-Neuve, Fidem, 1997, p. 109-131.

21. Morelle (Laurent), «Archives et histoire vers l'an mil. Une nouvelle " mutation " , Histoire et archives, 3, 1998, p. 119-141. [À propos de Patrick J. Geary, Mémoire et oubli à la fin du premier millénaire, Paris, Aubier, 1996 (Histoires), éd. orig. Phantoms of remembrance. Memory and oblivion at the end of the first millenium, Princeton, Princeton University Press, 1994.]

22. Guyotjeannin (Olivier), «La science des archives à Saint-Denis (fin du XIII ${ }^{\mathrm{e}}$-début du XVI ${ }^{\mathrm{e}}$ siècle) », in Autrand (Françoise), Gauvard (Claude) et MoEglin (Jean- 
Marie), éd., Saint-Denis et la royauté, études offertes à Bernard Guenée, Paris, Publications de la Sorbonne, 1999 (Histoire ancienne et médiévale, 59), p. 339-353.

23. HEIDECKER (Karl), éd., Charters and the use of the written word in medieval society, Turnhout, Brepols, 2000 (Utrecht Studies in Medieval Literacy, 5).

24. Guyotjeannin (Olivier), «La tradition de l'ombre. Les actes sous le regard des archivistes médiévaux (Saint-Denis, $\mathrm{XII}^{\mathrm{e}}-\mathrm{XV}^{\mathrm{e}}$ siècles) », in Kosto (Adam J.) et WinRoth (Anders), éd., Charters, cartularies and archives. The preservation and transmission of documents in the medieval West, proceedings of a colloquium of the Commission internationale de diplomatique, Princeton et New York, 16-18 sept. 1999, Toronto, Pontifical Institute of Medieval Studies, 2002 (Papers in Mediaeval Studies, 17), p. 81-112.

\subsubsection{Archives en France sous l'Ancien Régime}

25. Fréminville (Edme de La Poix de), Pratique universelle pour la rénovation des terriers et des droits seigneuriaux, Paris, chez Morel, 1746, in- $4^{\circ}, 2^{\mathrm{e}}$ éd. 5 vol., Paris, Knapen, 1752-1757.

26. Le MoIne (Pierre-Camille), Diplomatique pratique, ou traité de l'arrangement des archives et Trésor des chartes, ouvrage nécessaire aux commissaires à terriers, aux dépositaires de titres des anciennes seigneuries, Metz, Joseph Antoine, 1765, in-4․ [Doté d'un supplément : Le MoIne (Pierre-Camille) et BATTENEY (Joseph), Supplément à la «Diplomatique pratique» de M. Le Moine, contenant une méthode sûre pour apprendre à déchiffrer les anciennes écritures et arranger les archives, Paris, Despilly, 1772 , in- $4^{\circ}$.]

27. Chevrières (Jean-Guillaume de), Le Nouvel Archiviste, contenant une nouvelle méthode de ranger un chartrier, dont l'ordre chronologique est la base, auquel on a joint des calculs et tables pour aider à la supputation des tems [sic] nécessaires aux archivistes et à ceux qui s'adonnent à la chronologie, Paris, chez l'Auteur, 1775.

28. MARIÉE (G.), Traité des archives dans lequel on enseigne le moyen de faire revivre les anciennes écritures et la manière de procéder le plus simplement au pouillé général pour y donner suite un ordre simple, laconique et constant, lequel s'applique aussi aux bibliothèques, Paris, Mariée, Cailleau, 1779.

29. Aubry de SAint-Vibert (Charles-Louis), Les Terriers rendus perpétuels ou mécanisme de leur confection (...), Paris, chez l'Auteur, 1787, in-fol.

30. Michel De Boislisle (Arthur), « Jean Du Tillet et le Trésor des chartes », AnnuaireBulletin de la Société de l'histoire de France, 1873, p. 106-111.

31. Outrey (Amédée), « Sur la notion d'archives en France à la fin du XVIII ${ }^{\mathrm{e}}$ siècle », Revue historique de droit français et étranger, $4^{\mathrm{e}}$ sér., 31e année, 1953, p. 277-286.

32. Outrey (A.), «La notion traditionnelle de titres et les origines de la législation révolutionnaire sur les archives, la loi du 7 septembre $1790 »$, Revue historique de droit français et étranger, $4^{\mathrm{e}}$ sér., 33e année, 1955, p. 438-463.

33. BAUdot (Marcel), « Les archives municipales dans la France de l'Ancien Régime », Archivum, vol. XIII, 1963, p. 23-59.

34. SANDRI (Leopoldo), La Letteratura archivistica dei secc. XVII-XVIII (Fonti e problemi), Naples, Archivio di Stato di Napoli. Scuola di Paleografia, 1961.

35. Nortier (Michel), « Le sort des archives dispersées de la Chambre des comptes de Paris », Bibliothèque de l'École des chartes, t. CXXIII, 1965, p. 460-537. 
36. BAUTIER (Robert-Henri), «La phase cruciale de l'histoire des archives. La constitution des dépôts d'archives et la naissance de l'archivistique, XVI $\mathrm{e}^{\mathrm{e}}$-début du XIX ${ }^{\mathrm{e}}$ siècle », Archivum, vol. XVIII, 1968, p. 139-150.

37. Pomian (Krzysztof), «Les historiens et les archives dans la France du XVII ${ }^{\mathrm{e}}$ siècle », Acta Poloniae Historica, vol. XXVI, 1972, p. 109-125.

38. HildesheImER (Françoise), «Archives et archivaires du pays de Provence », Provence historique, t. XXXV, 1975, p. 587-598.

39. Gembicki (Daniel), Histoire et politique à la fin de l'Ancien Régime. Jacob-Nicolas Moreau, 1717-1783, Paris, A. G. Nizet, 1979.

40. Barret-Kriegel (Blandine), Les Historiens et la monarchie, 4 vol., Paris, Presses universitaires de France, 1988, repr. in KRIEGEL (B.), L'Histoire à l'âge classique. Vol. I : Jean Mabillon; vol. II : La défaite de l'érudition; vol. III : Les académies de l'histoire; vol. IV : La République incertaine, Paris, Presses universitaires de France, 1996 (Quadrige, 231-234).

41. Deaux (Christiane), "Rénover un terrier en Lyonnais au XVIII ${ }^{\mathrm{e}}$ siècle », Revue historique de droit français et étranger, 72e année, 1994, p. 367-382.

42. BÉCHU (Philippe), Un feudiste et ses clients à la veille de la Révolution, in Plaisirs d'archives, recueil de travaux offerts à Danièle Neirinck, Mayenne, Impr. de la Manutention, 1997, p. 191-234.

43. Brown (Elizabeth A. R.), « Jean Du Tillet, François $\mathrm{I}^{\mathrm{er}}$ and the Trésor des chartes », in Histoire d'archives, recueil de travaux offerts à Lucie Favier par ses collègues et amis, Mayenne, Impr. de la Manutention, 1997, p. 237-247.

44. Brown (E. A. R.), « Jean Du Tillet et les Archives de France », Histoire et archives, 2, 1997, p. 29-63.

45. Collin (Hubert), Le Trésor des chartes de Lorraine, ses lieux de conservation successifs et les amoindrissements qu'il a subis aux XVII et XVIII ${ }^{e}$ siècles, in Plaisirs d'archives, recueil de travaux offerts à Danièle Neirinck, Mayenne, Impr. de la Manutention, 1997, p. 247-276.

\subsubsection{Archives en France après 1789}

46. BORDIER (Henri), Les Archives de la France ou histoire des archives de l'Empire, des archives des ministères, des départements, des communes, des hôpitaux, des greffes, des notaires, etc., contenant l'inventaire de ces dépôts, Paris, Dumoulin, 1855, repr. Genève, Megariotis Reprints, 1978.

47. LABORDE (Léon de), «Les archives de France pendant la Révolution », [introduction à] Inventaire du fonds d'archives dit «Les monuments historiques », Paris, impr. J. Claye, 1866, in- $4^{\circ}$, p. I-CXIV, $2^{\mathrm{e}}$ éd. sous le titre Les Archives de France, leurs vicissitudes pendant la Révolution, leur régénération sous l'Empire, Paris, Veuve Renouard, 1867, in-12.

48. BOUTARIC (Edgard), «Le vandalisme révolutionnaire, les archives pendant la Révolution française », Revue des questions historiques, t. XII, 1872, p. 325-396.

49. LoKke (Carl L.), "Archives and the French Revolution », American Archivist, vol. XXXI, 1968, p. 23-31.

50. Ducrot (Ariane), «Comment fut élaborée et votée la loi sur les archives du 3 janvier 1979 », La Gazette des archives, nouv. sér., 104, 1979, p. 17-41. 
51. Chomel (Vital), «Les titres de la féodalité et la mémoire de la nation », in ChiAnEA (Gérard), dir., Les Droits de l'homme et la conquête des libertés. Des Lumières aux révolutions de 1848, actes du colloque de Grenoble-Vizille, 1986, Grenoble, Presses universitaires de Grenoble, 1988, p. 314-325.

52. Archives et Révolution. Création ou destruction?, actes du colloque de Châteauvallon (Var), 10-11 mars 1988, La Gazette des archives, nouv. sér., nº spéc., 146-147, 1989.

53. Delmas (Bruno), Archives, mémoire et système politique en France au XIXe siècle, in Espagne (Michel), Middel (Katharina) und Middel (Matthias), Hrsg., Archiv und Gedächtnis. Studien zur interkulturellen Überlieferung, Leipzig, Leipziger Universitäts-Verlag, 2000 (Deutsch-Französich Kulturbibliothek, 13), p. 37-49.

54. HiLDESHEIMER (Françoise), «Une politique pour les archives, 1880-1940? », in BARUCH (Marc-Olivier) et DuClERT (Vincent), dir., Serviteurs de l'État. Une histoire politique de l'administration française, 1875-1945, Paris, La Découverte, 2000, p. 373-386.

\section{ARCHIVISTIQUE}

\subsection{Généralités}

\subsubsection{Bibliographie}

55. DUCHEIN (Michel), Bibliographie internationale fondamentale d'archivistique, New York-Londres-Paris, K. G. Saur, 1978 (Archivum, XXXV). [Très peu de titres antérieurs à 1950.]

\subsubsection{Revues}

56. La Gazette des archives: revue trimestrielle, anc. sér. 1933-1939, nouv. sér. depuis 1947 - tables cumulatives des années 1933-1984 publiées en 1986, tables de 1933 à 1999 disponibles sur www.archivistes.org [Émanation de l'Association des archivistes français.]

57. Archivum : revue annuelle, 1951-2000. [Remplacé par Comma. Émanation du Conseil international des Archives.]

58. Janus: revue trimestrielle, 1989-2000. [Remplacé par Comma. Émanation du Conseil international des Archives.]

59. Comma. Revue internationale d'archivistique : revue trimestrielle, depuis 2001. [Émanation du Conseil international des Archives.]

\subsection{Ouvrages et manuels}

60. Champollion-Figeac (Aimé), Les Archives départementales de France. Manuel de l'archiviste des préfectures, des mairies et des hospices, Paris, Dupont, 1860.

61. Richou (Gabriel), Traité théorique et pratique des archives publiques, Paris, Dupont, 1883 (Répertoire du droit administratif). 
62. Desjardins (Gustave), Le Service des archives départementales. Conférences faites aux élèves de l'École des chartes les 10, 18, 25 et 30 juin 1890, Paris, E. Bourloton, 1890.

63. Manuel d'archivistique. Théorie et pratique des archives publiques en France, Paris, SEVPEN, 1970.

64. Favier (Jean) et NeIRINCK (Danièle), dir., La Pratique archivistique française, Paris, Archives nationales, 1994.

\subsection{Respect des fonds et traitement des archives}

65. Celier (Léonce), « La responsabilité de l'archiviste dans l'élimination des papiers inutiles », La Gazette des archives, 7, 1950, p. 9-14. [Texte d'une conférence donnée à la Société de l'École nationale des chartes le 22 décembre 1949 par un inspecteur général des Archives.]

66. Lodolini (Elio), « Identificazione dell'archivio », Rassegna degli Archivi di Stato, t. XVIII, 3, 1958, p. 306-321. [Un fonds d'archives est d'abord un ensemble clos, une universitas rerum: partant, l'auteur dénie cette qualité aux documents administratifs contemporains.]

67. POSNER (Ernst), « Max Lehmann and the genesis of the " principle of provenance " », Indian Archives, 4, 1950, p. 133-141, réimpr. in ID., Archives and the public interest. Selected essays, éd. Kenneth Munden, Washington, Public Affairs Press, 1967, p. 36-44.

68. PAPRITZ (Johannes), « Neuzeitlichen Methoden der archivistichen Ordnung (Schriftgut vor 1800)», Archivum, t. XIV, 1964, p. 13-56. [Du classement à l'inventaire, examen des divers principes archivistiques fondamentaux.]

69. BoISARD (Pierre), « Pour une politique des éliminations ? Réflexions sur la pratique des Archives de la Seine », La Gazette des archives, nouv. sér., 59, 1967, p. 205-238.

70. «Problèmes de structure et d'inventaire (à propos d'un article récent, à quatre voix, avec des sous-titres pour chacun des auteurs) », La Gazette des archives, nouv. sér., n spéc., 60, 1968, p. 13-30 (I. Pierre CAILLET, « Ouverture pour un dialogue », p. 13-15 ; II. Rémi MATHIEU, «La série F des Archives nationales », p. 15-19; III. Jean WAQUET, « Structures de classement, éliminations de documents, élaboration de répertoires dans les Archives nationales et départementales », p. 20-24 ; IV. Robert FAVREAU, «Deux conceptions différentes », p. 25-30). [À propos de l'article de Robert FAVREAU, « Une expérience de collaboration entre l'Administration et les Archives. Bilan et réflexions », La Gazette des archives, nouv. sér., 57, 1967, p. 117-124, et de l'inadaptation archivistique et administrative de la série $\mathrm{F}$ des Archives nationales.]

71. LAROCHE (Carlo), «Que signifie le respect des fonds ? Esquisse d'une archivistique structurale », La Gazette des archives, suppl., 73, 1971.

72. Duchein (Michel), «La clef du trésor. L'évolution des instruments de recherche d'archives du Moyen Âge à nos jours d'après des exemples français », in ID., Études d'archivistique 1957-1992, Paris, Association des archivistes français, 1992, p. 9-34.

73. Duchein (M.), «Le respect des fonds en archivistique. Principes théoriques et problèmes pratiques », La Gazette des archives, 97, 1977, p. 71-96, réimpr. in ID., 
Études d'archivistique 1957-1992, Paris, Association des archivistes français, 1992, p. 9-34. [À noter la dernière partie de l'article, p. 95-96, « IV. L'instrument de recherche archivistique comme moyen de remédier aux difficultés du respect des fonds ».]

74. Aubry (Marie-Thérèse), LANGlois (Monique) et Reydellet (Chantal), «Les parlements de France et leurs archives », La Gazette des archives, nouv. sér., 125-126, 1984, p. 125-143.

75. Charon-Bordas (Jeannine), «Les archives des assemblées nationales. Exemple de traitement d'un fonds moderne », La Gazette des archives, nouv. sér., 125-126, 1984, p. 144-155.

76. Guyotjeannin (Olivier), «Tris et échantillonnages. Empirisme et théorie », La Gazette des archives, nouv. sér., 124, 1984, p. 5-24. [Article de théorie, fruit d'un travail de réflexion sur les archives contemporaines françaises.]

77. Collin (Hubert), «La mise en œuvre du principe de respect des fonds. Réflexion sur l'état actuel », Archives, t. XXII, 2, automne 1990, p. 3-8.

78. HiLDESHEIMER (Françoise), «Des triages au respect des fonds. Les archives en France sous la Monarchie de Juillet », Revue historique, t. CCLXXXVI/2, 580, 1991, p. 295-312.

79. «Fonds judiciaires et recherche historique », La Gazette des archives, nouv. sér., nº spéc., 158-159, 1992.

80. BASTIEN (Hervé), «La normalisation du processus de tri », Archivum, t. XXXIX, 1994, p. 222-234.

81. Lodolini (Elio), « Respect des fonds et principe de provenance. Histoire, théories et pratiques », La Gazette des archives, 168, 1995, p. 201-212.

82. GuYOtJEANNin (Olivier), «Les méthodes de travail des archivistes du roi de France (fin XIII ${ }^{\mathrm{e}}$-début XVI ${ }^{\mathrm{e}}$ siècle) », Archiv für Diplomatik, t. XLII, 1996, p. 295-373.

83. Carvais (Robert), « La classification des actes du fonds judiciaire de la Chambre des bâtiments. Une application à l'époque moderne de la hiérarchie postrévolutionnaire des pouvoirs », Histoire et archives, t. IV, 1998, p. 31-130. [Classement archivistique et classification juridique : un décalage.]

84. Descamps (Florence), L'Historien, l'archiviste et le magnétophone. De la constitution de la source orale à son exploitation, Paris, Comité pour l'histoire économique et financière de la France, 2001 (Histoire économique et financière de la France. Sources).

\subsection{Confection des instruments de recherche}

85. LuCE (Siméon), «De l'utilité matérielle et pratique, de l'importance historique et scientifique, de la portée morale et sociale des travaux d'archives à propos d'un discours de Son Excellence M. le comte de Persigny », Bibliothèque de l'École des chartes, t. XXIV, 1863, p. 237-251.

86. BORDIER (Henri), Les Inventaires des archives de l'Empire. Réponse à Monsieur le marquis de Laborde, directeur général, contenant un errata pour ses préfaces et ses inventaires, Paris, Bachelin et Deflorenne, 1867, in- $4^{\circ}$.

87. PANNIER (Léopold), «État des inventaires sommaires et des autres travaux relatifs aux diverses archives de la France au $1^{\mathrm{er}}$ janvier 1875 », Bibliothèque de l'École des chartes, t. XXXVI, 1875, p. 5-80. [Bilan d'un quart de siècle de réalisation d'instruments de recherche depuis la circulaire de 1841.] 
88. «Instruction du 25 mars 1909 sur la rédaction de l'inventaire sommaire et du répertoire numérique dans les archives départementales ", in Lois, décrets, arrêtés, règlements et instructions concernant le service des archives départementales, $2^{\mathrm{e}}$ éd. Melun, Imprimerie administrative, 1931, p. 149-170.

89. MAhiEU (Bernard), «Les inventaires d'archives selon Michelet », La Gazette des archives, 16, 1954, p. 16-22.

90. Monicat (Jacques), «Les archives notariales en France. Utilisation scientifique », La Gazette des archives, 40, 1963, p. 16-24.

91. «Les archives et la recherche », $n^{\circ}$ spéc. de La Gazette des archives, nouv. sér., 53, 1966. [Dialogue concret des archivistes et des chercheurs sur les instruments de recherche et la communication des archives: réalisations et espoirs des premiers, études, attentes et insatisfactions des seconds. En particulier :

«La recherche et les archives nationales », p. 89-121 (exposés de Pierre Timbal, p. 90-93, Albert Mirot, p. 93-98, Pierre Renouvin, p. 98-105, Pierre Caillet, p. 105-115, Bernard MAHIEU, p. 115-121);

« La recherche et les archives départementales », p. 123-140 (exposés de Jean SCHNEIDER, p. 123-126, Jean VIDALENC, p. 126-129, François BuRCKARD et François HIMLY, p. 129-140).]

92. HILDESHEIMER (Françoise), «Une archivistique des manques? », Bibliothèque de l'École des chartes, t. CXXXVIII, 1980, p. 231-235.

93. Hildesheimer (F.), «Inventaire d'archives et recherche historique », La Gazette des archives, nouv. sér., 117-118, 1982, p. 71-81.

94. Chanaud (Robert) et Dubois (Martine), «Comment peut-on classer des fonds anciens aujourd'hui ? », La Gazette des archives, nouv. sér., 125-126, 1984, p. 212-231.

95. HILDESHEIMER (Françoise), « Fonds fermés, fonds morts ? », La Gazette des archives, nouv. sér., 125-126, 1984, p. 257-259.

96. VAlléE (Aline), «Index thématique, tableaux méthodiques et thésaurus. Luxe ou nécessité ? », La Gazette des archives, nouv. sér., 125-126, 1984, p. 232-248.

97. DuCHEIN (Michel), «La clef du trésor. L'évolution des instruments de recherche d'archives du Moyen Âge à nos jours d'après des exemples français », in Miscellanea Carlos Wyffels, $\mathrm{n}^{\circ}$ spéc. d'Archives et bibliothèques de Belgique, t. LVII, 1986, p. 109-126.

98. Bimbenet-Privat (Michèle), «Les répertoires numériques rédigés aux Archives nationales vers 1890. Le travail d'une génération », La Gazette des archives, nouv. sér., 152-153, 1991, p. 48-54.

99. Cavillon (Céline), «Les inventaires du Trésor des chartes et de la Chambre des comptes de Paris à l'époque moderne », La Gazette des archives, nouv. sér., 166, 1994, p. 285-303.

100. Delaume-Boutet (Liliane), "Le chartrier de l'évêché de Limoges, cotation et inventaires », Bibliothèque de l'École des chartes, t. CLII, 1994, p. 159-203.

101. HILDESHEIMER (Françoise), «Les premières publications des archives », in Bercé (Yves-Marie) et Contamine (Philippe), dir., Histoire de France, historiens de la France, actes du colloque international, Reims, 14 et 15 mai 1993, Paris, Honoré Champion, 1994, p. 281-299.

102. HiLdesheimer (F.), « De l'inventaire des layettes du Trésor des chartes au Guide des sources de la Seconde Guerre mondiale », in Les Instruments de recherche et 
leurs utilisateurs. Bilan et perspectives, journées d'étude de la direction des Archives de France, Le Mans, 28-29 sept. 1995, Paris, Direction des Archives de France, 1996, p. 9-23.

103. NOUgARET (Christine), « De Nathalis de Wailly à MIRA. 150 ans de normalisation des instruments de recherche aux Archives nationales », in Histoire d'archives, recueil d'articles offerts à Lucie Favier par ses collègues et amis, Paris, Société des amis des Archives de France, 1997, p. 85-104.

104. NOUGARET (Christine) et GALland (Bruno), Les Instruments de recherche dans les archives, Paris, La Documentation française, 1999.

\section{3. ÉPISTÉMOLOGIE, HISTORIOGRAPHIE ET ARCHIVES}

\subsection{Numéros spéciaux de revues}

105. « L'archive », nº spéc., Traverses, t. XXXVI, 1986.

106. «Archives et anthropologie », n spéc., Gradhiva, 30-31, déc. 2001.

107. «Les historiens et les archives, table ronde de la Société d'histoire moderne et contemporaine, 31 mars 2001 », Revue d'histoire moderne et contemporaine, t. XLVIII, 4bis, suppl. 2001.

\subsection{Autres travaux}

108. Moreau (Jacob-Nicolas), Plan des travaux littéraires ordonnés par Sa Majesté pour la recherche, la collection et l'emploi des monuments de l'histoire et du droit public de la monarchie française, Paris, Imprimerie royale, 1782.

109. LANGLoIs (Charles-Victor) et SEIGNobos (Charles), Introduction aux études historiques, Paris, Hachette, 1898, réimpr. Paris, Kimé, 1992.

110. Foucault (Michel), L'Archéologie du savoir, Paris, Gallimard, 1969 (Bibliothèque des sciences humaines).

111. FurET (François), «L'histoire quantitative et la construction du fait historique », Annales E.S.C., 26, 1971, p. 63-75, repr. in LE GoFf (Jacques) et NorA (Pierre), dir., Faire de l'histoire, 3 vol., Paris, Gallimard, 1974 (Bibliothèque des histoires), vol. I, p. 42-61.

112. CERTEAU (Michel de), L'Écriture de l'histoire, Paris, Gallimard, 1975 (Bibliothèque des histoires), réimpr. Paris, Gallimard, 2002 (Folio Histoire, 115).

113. ChOMEl (Vital), « Une autre archivistique pour une nouvelle histoire », La Gazette des archives, nouv. sér., 88, 1975, p. 238-248. [Réaction d'un archiviste à la publication des trois volumes de Faire de l'histoire ; sur le décalage entre le tournant historiographique pris dans les années 1930 et la pratique archivistique à l'époque de l'article.]

114. Krakovitch (Odile), «Des archives pour quelle histoire ? (le débat n'est pas clos) », La Gazette des archives, nouv. sér., 96, 1977, p. 23-31. [Le plaidoyer d'une archiviste en faveur des publics autres que les universitaires et chercheurs «professionnels».]

115. GuENÉE (Bernard), Histoire et culture historique dans l'Occident médiéval, Paris, Aubier-Montaigne, 1980 (Collection historique). 
116. FINLEY (Moses I.), « Documents », in ID., Ancient history. Evidence and models, Londres, Chatto and Windus, 1985, p. 27-46, trad franç. Jeannie CARLIER, « Le document », in ID., Sur l'histoire ancienne. La matière, la forme et la méthode, Paris, La Découverte, 1987 (Textes à l'appui. Histoire classique), p. 68-96.

117. Melot (Michel), « Des archives considérées comme substances hallucinogènes », Traverses, t. XXXVI, 1986, p. 14-19.

118. FARGe (Arlette), Le Goût de l'archive, Paris, Seuil, 1989, rééd. Paris, Seuil, 1997 (Points Histoire, 233).

119. OphIR (Adi), « Des ordres dans l'archive », Annales E.S.C., 45e année, 3, 1990, p. 735-754. [Sur la notion d' « archive » et l'ordonnancement du discours historique auquel elle donne lieu. La «fabrication des archives » en tant que telle n'est pas envisagée.]

120. Muchembled (Robert), «Un historien aux archives. Itinéraire d'une recherche dans les fonds d'Ancien Régime », La Gazette des archives, nouv. sér., 158-159, 1992, p. 241-248.

121. HILDESHEIMER (Françoise), « Fonds d'archives et histoire religieuse. Un décalage ? », La Gazette des archives, nouv. sér., 165, 1994, p 220-222.

122. Chabin (Marie-Anne), Je pense donc j'archive. L'archive dans la société de l'information, Paris, L'Harmattan, 1999.

123. DANCEL (Brigitte), «La place du document dans la nouvelle didactique de l'histoire », La Gazette des archives, nouv. sér., 184-185, 1999, p. 95-103.

124. Monnier (François), «De l'intérêt historique. À propos d'un avant-projet de loi sur les archives », Revue administrative, 309, 1999, p. 227-229. [Contre les «prétentions des archivistes » à procéder seuls à des tris pour ne conserver que les documents présentant un « intérêt historique ».]

125. MüLLER (Bertrand), «Des archives en mutation et du vertige de l'historien. Remarques historiographiques », Studien und Quellen. Études et sources, t. XXVII, 2001, p. 49-63.

126. Pomian (Krzysztof), « Du monopole de l'écrit au répertoire illimité de sources. Un siècle de mutations de l'histoire», Studien und Quellen. Études et sources, t. XXVII, 2001, p. 15-33.

127. Ricceur (Paul), La Mémoire, l'histoire, l'oubli, Paris, Seuil, 2000 (L'Ordre philosophique), réimpr. Paris, Seuil, 2003 (Points Essais, 494).

\section{Olivier PONCET*, Centre historique des Archives nationales, École nationale des chartes, 60 , rue des Francs-Bourgeois, F-75141 Paris Cedex 03, olivier.poncet@culture.gouv.fr} (2003).

\footnotetext{
* Olivier PonCET, né en 1969, conservateur du patrimoine, professeur associé à l'École nationale des chartes. Ses travaux portent sur la diplomatique des actes et la sociologie des institutions françaises et pontificales à l'époque moderne.
} 


\section{INDEX DES NOMS D'AUTEURS ET DE REVUES}

Archivum : 57

AUBRY (Marie-Thérèse) : 74

Aubry dE SAINT-VIBERT (Charles-Louis) : 29

BARRET-KRIEGEL (Blandine) : 40

BASTIEN (Hervé) : 80

BAUdoT (Marcel) : 33

BAUTIER (Robert-Henri) : 4, 36

BÉCHU (Philippe) : 42

Bimbenet-Privat (Michèle) : 98

BOISARD (Pierre) : 69

BORDIER (Henri) : 46, 86

BOUTARIC (Edgard) : 48

Brown (Elizabeth A. R.) : 43, 44

BurCKARD (François) : 91

Caillet (Pierre) : 70, 91

Carvais (Robert) : 83

Cavillon (Céline) : 99

Celier (Léonce) : 65

Certeau (Michel de) : 112

Chabin (Marie-Anne) : 122

Champollion-Figeac (Aimé) : 60

Chanaud (Robert) : 94

Charon-Bordas (Jeannine) : 75

Châteauvallon (colloque) : 52

Chevrières (Jean-Guillaume de) : 27

Chomel (Vital) : 51, 113

CEuré (Sophie) : 7

Collin (Hubert) : 45, 77

Comma : 59

Contamine (Philippe) : 17, 19, 101

D'AdDARIO (Arnaldo) : 5

DANCEL (Brigitte) : 123

DEAUX (Christiane) : 41

DELABORDE (Henri François) : 14

Delaume-Boutet (Liliane) : 100

Delmas (Bruno) : 53

Demougin (Ségolène) : 10

DESCAMPS (Florence) : 84

DESJARDINS (Gustave) : 62

DuboIs (Martine) : 94

DuCHEIN (Michel) : 55, 72, 73, 97

DUCLERT (Vincent) : 7, 54
DuCRot (Ariane) : 50

FARGE (Arlette) : 118

FAVIER (Jean) : 3, 64

FAVREAU (Robert) : 70

FINLEY (Moses I.) : 116

Foucault (Michel) : 110

FréminviLle (Edme de La Poix de) : 25

FurET (François) : 111

Galland (Bruno) : 104

Gazette des archives (La) : 56

GEMBICKI (Daniel) : 39

GoOdy (Jack) : 9

GUENÉE (Bernard) : 115

GuyotjEannin (Olivier) : 20, 22, 24, 76, 82

HEIDECKER (Karl) : 23

HILDESHEIMER (Françoise) : 13, 38, 54, 78, $92,93,95,101,102,121$

HimLY (François) : 91

Histoire et archives : 6

Janus : 58

KraKOVITCH (Odile) : 114

LABORDE (Léon de) : 47

LANGLOIS (Charles-Victor) : 109

LANGLOIS (Monique) : 74

LAROCHE (Carlo) : 71

Le MoIne (Pierre-Camille) : 26

Lodolini (Elio) : 66, 81

LOKKE (Carl L.) : 49

LuCE (Siméon) : 85

MAHIEU (Bernard) : 89, 91

Manuel d'archivistique : 63

MARIÉE (G.) : 28

Mathieu (Rémi) : 70

MAttÉONi (Olivier) : 19

Melot (Michel) : 117

Michel DE Boislisle (Arthur) : 30

Mirot (Albert) : 91

Monicat (Jacques) : 90

MonNIER (François) : 124

Moreau (Jacob-Nicolas) : 108

Morelle (Laurent) : 21

Muchembled (Robert) : 120 
MÜLLER (Bertrand) : 125

NEIRINCK (Danièle) : 64

NORTIER (Michel) : 35

Nougaret (Christine) : 103, 104

OPHIR (Adi) : 119

Outrey (Amédée) : 31, 32

PANNIER (Léopold) : 87

PAPRITZ (Johannes) : 68

Pistolese (Serafino) $: 1$

Pomian (Krzysztof) : 12, 37, 126

POSNER (Ernst) : 8, 67

RENOUVIN (Pierre) : 91

REYDELLET (Chantal) : 74
RICHARD (Jean) : 15

RICHOU (Gabriel) : 61

RICEUR (Paul) : 127

RÜCK (Peter) : 16

SANDRI (Leopoldo) : 2, 34

SCHNEIDER (Jean) : 91

Seignobos (Charles) : 109

SICKINGER (James P.) : 11

Sот (Michel) : 18

TIMBAL (Pierre) : 91

VALLÉE (Aline) : 96

VIDALENC (Jean) : 91

WAQUET (Jean) : 70 